\title{
Runge-Kutta and Block by Block Methods to Solve Linear Two- Dimensional Volterra Integral Equation with Continuous Kernel
}

\author{
A. M. Al-Bugami ${ }^{1}$, M. M. Al-Wagdani ${ }^{2}$ \\ ${ }^{1}$ Department of Mathematics, Faculty of Sciences Taif University, KSA \\ ${ }^{2}$ Department of Mathematics, Faculty of Sciences Taif University, KSA
}

Abstract: In this paper, the existence and uniqueness of solution of the linear two dimensional Volterra integral equation of the second kind with Continuous Kernel are discussed and proved.RungeKutta method(R. KM)and Block by block method (B by BM) are used to solve this type of two dimensional Volterra integral equation of the second kind. Numerical examples are considered to illustrate the effectiveness of the proposed methods and the error is estimated.

Keywords: Two-Dimensional Volterra Integral Equation; Runge-Kutta method; Block- by -block method.

\section{Council for Innovative Research}

Peer Review Research Publishing System

Journal: JOURNAL OF ADVANCES IN MATHEMATICS

Vol .11, No. 5

www.cirjam.com , editorjam@gmail.com 


\section{INTRODUCTION:}

Two- dimensional integral equations provide an important tool for modeling many problems in engineering and science [1]. There are many well-written texts on the theory and applications of integral equations in different sciences. From 1960to the present day, many new numerical methods have been developed for the solution of many types of integral equations, such as the Toeplitz matrix method, the product Nyström method, the Galerkin method; R. KM and B by BM (see Linz [2], Baker et al. [3], and Delves and Mohamed [4]). More information for some numerical methods can be found especially in Delves and Mohamed [4], Atkinson [5, 6] and Golberg [7]. In the reference [8], B by BM was used to solve the integral equation in one dimensional. In [9], the authors solved the TD-NIE of the second kind using degenerate kernel method. Guoqiang et al., in [10], obtained numerically the solution of two-dimensional nonlinear Volterra integral equation by collocation and iteration collocation methods. In [11], Guoqiang and Jiong analyzed the existence of asymptotic error expansion of the Nyström solution for two-dimensional nonlinear Fredholm integral equation of the second kind. In [12], Abdou obtained, using separation variables method, the solution of the linear F-VIE in one, two and three dimensional.In [13],El-Kalla and Al-Bugami solved nonlinear two-dimensional Volterra integral equation by using Adomian and Block by block methods. In this paper, we use R. KM and B by BM to discuss numerically the solution of the LT-DVIE of the second kind with continuous kernel of the form

$\mu u(x, y)=f(x, y)+\lambda \int_{0}^{x} \int_{0}^{y} k(x, y, t, s) u(t, s) d t d s$

where $\mu$ is a constant defines the kind of the integral equation, $u(x, y)$ is an unknown function, will be determined, the functions $f(x, y)$ and $k(x, y, t, s)$ are given analytical functions defined, respectively, $J=[0, \mathrm{X}] \times[0, \mathrm{Y}]$, $E=\{(x, y, t, s): 0 \leq t \leq x \leq a, 0 \leq s \leq y \leq b\}, \mu$ and $\lambda$ are constants that have many physical meanings.

\section{The existence and uniqueness of the solution:}

To guarantee the existence of a unique solution of equation (1), we assume the following conditions:

(i) The kernel $k(x, y, t, s)$ is continuous function in $E$ satisfies:

$|k(x, y, t, s)| \leq K \quad, K$ is a constant

(ii) The given function $f(x, y)$ is continuous with its derivatives and belongs to $J=[0, \mathrm{X}] \times[0, \mathrm{Y}]$, and its norm is defined as:

$\|f(x, y)\|=\max _{x, y \in J}|f(x, y)| \leq M \forall(x, y) \in J \quad, M$ is a constant.

(iii) the unknown function $u(x, y)$ is satisfies the Lipschits condition with respect to its argument and its normal is defined in $L_{2}[0, X] \times L_{2}[0, Y]$ as:

$\|u(x, y)\|=\left[\int_{0}^{x} \int_{0}^{y}|u(x, y)|^{2} \mathrm{dxdy}\right]^{1 / 2} \leq C \quad$, where $C$ is a constant (2)

To prove the existence a unique solution of (1) using Banach fixed point theorem. Rewrite equation (3.1) in the integral operator form:

$\bar{T} u(x, y)=\frac{1}{\mu} f(x, y)+T u(x, y)(3)$

Where

$T u(x, y)=\frac{\lambda}{\mu} \int_{0}^{x} \int_{0}^{y} k(x, y, t, s) u(t, s) d t d s(4)$

\section{Theorem 1:}

If the conditions (i), (ii) and (iii) are verified, then equation (1) has auneque solution in the Banach space $C([0, \mathrm{X}] \times[0, \mathrm{Y}])$, the proof of tis theorem depends on the following two lemmas.

\section{Lemma 1:}


Underthe conditions (i)-(iii), the operator $\bar{T}$ defined by (3), maps the space $C([0, \mathrm{X}] \times[0, \mathrm{Y}])$ into itself.

\section{Proof:}

In view of the formulas (3) and (4), then using the condition (ii), and applying Couchy-Shwarz inequality, we have

$\|\bar{T} u(x, y)\| \leq \frac{1}{|\mu|}\|f(x, y)\|+\left|\frac{\lambda}{\mu}\right|\left\|\left|\int_{0}^{x} \int_{0}^{y}\right| k(x, y, t, s)\right\| u(t, s)|d t d s|$

Using the condition (i) and (iii), the above inequality takes the form:

$\|\bar{T} u(x, y)\| \leq \frac{M}{|\mu|}+A\|u(x, y)\| \quad, A=\left|\frac{\lambda}{\mu}\right| K C$

Inequality (6) show that, the operator $\bar{T}$ maps the space $C([0, \mathrm{X}] \times[0, \mathrm{Y}])$ into itself.

Moreover, the inequality (6) involves that the operator $T$ is bounded where

$$
\|T u(x, y)\| \leq A\|u(x, y)\|
$$

The inequalities (6) and (7) define that the operator $\bar{T}$ is bounded.

\section{Lemma 2:}

If the conditions (i) and (iii) are satisfied then the operator $\bar{T}$ is contractive in the Banach space $C([0, \mathrm{X}] \times[0, \mathrm{Y}])$.

\section{Proof:}

For the two functions $u_{1}(x, y)$ and $u_{2}(x, y)$ in the space $C([0, \mathrm{X}] \times[0, \mathrm{Y}])$ the formula (3) and (4) lead to,

$$
\left\|\left(\bar{T} u_{1}-\bar{T} u_{2}\right)(x, y)\right\| \leq\left|\frac{\lambda}{\mu}\right||| \int_{0}^{x} \int_{0}^{y}|k(x, y, t, s)|\left|u_{1}(t, s)-u_{2}(t, s)\right| d t d s \|
$$

Using the condition (iii) and then applying Cauchy-Shwarz inequality, we have

$$
\left\|\left(\bar{T} u_{1}-\bar{T} u_{2}\right)(x, y)\right\| \leq A\left\|u_{1}(t, s)-u_{2}(t, s)\right\|
$$

Inequality (8) show that, the operator $\bar{T}$ is continuous in the space $C([0, \mathrm{X}] \times[0, \mathrm{Y}])$.

Also, $\bar{T}$ is a contraction operator, under the condition $A<1$, in the Banach space $C([0, \mathrm{X}] \times[0, \mathrm{Y}])$. Therefore, the operator $\bar{T}$ has a unique fixed point which is the unique solution of equation (1).

\section{Runge-Kutta method:}

In this section, the R. KM is used to solve linear two-dimensional Volterra integral equation of the second kind:

$$
u(x, y)=f(x, y)+\lambda \int_{0}^{x} \int_{0}^{y} k(x, y, t, s) u(t, s) d t d s
$$

We introduce the intermediate values,

$$
U_{n p}=F_{n}\left(x_{n}+\theta_{p} h, y_{n}+\theta_{p} h\right)+h \sum_{q=0}^{p-1} A_{p q} k\left(x_{n}+d_{p q} h, y_{n}+d_{p q} h, x_{n}+c_{q} h, y_{n}+c_{q} h\right) U_{n q}
$$


For $p=1,2, \ldots m$. Using $F(0,0)=U_{00}=f(0,0)$, the equation (10) can be used to determine the sequence of values $U_{00}, U_{01}, \ldots, U_{0 m}, U_{10}, U_{11}, \ldots, U_{1 m}, \ldots$, the value $U_{n m}$ is taken as an approximation to $u\left(x_{n}+h, y_{n}+h\right)$ ,also $U_{n m}=U_{n+1,0}$.

The function $F_{n}(x, y)$ is chosen so that it is an approximation to

$f(x, y)+\int_{0}^{x_{n}} \int_{0}^{y_{n}} k(x, y, t, s) u(t, s) d t d s$

While the parameters $\theta_{p}, A_{p q}, d_{p q}, c_{q}$ are determined by a Taylor expansion. For example, by setting $\theta_{p}=d_{p q}=c_{p}$, we obtain a set of formulas were called Pouzet-type.

For $m=4$, a particular set of parameters for a Pouzet-type method is

$$
\begin{aligned}
& \theta_{0}=0, \quad \theta_{1}=\theta_{2}=\frac{1}{2}, \quad \theta_{3}=\theta_{4}=1 \\
& A_{10}=A_{21}=\frac{1}{2}, \quad A_{20}=A_{30}=A_{31}=0, \quad A_{32}=1 \\
& A_{40}=A_{43}=\frac{1}{6}, \quad A_{41}=A_{42}=\frac{1}{3}
\end{aligned}
$$

Then, the system (10) becomes,

$$
\begin{aligned}
& U_{n 0}=F_{n}\left(x_{n}, y_{n}\right) \\
& U_{n 1}=F_{n}\left(x_{n}+\frac{h}{2}, y_{n}+\frac{h}{2}\right)+\frac{h}{2} k\left(x_{n}+\frac{h}{2}, y_{n}+\frac{h}{2}, x_{n}, y_{n}\right) U_{n 0} \\
& U_{n 2}=F_{n}\left(x_{n}+\frac{h}{2}, y_{n}+\frac{h}{2}\right)+\frac{h}{2} k\left(x_{n}+\frac{h}{2}, y_{n}+\frac{h}{2}, x_{n}+\frac{h}{2}, y_{n}+\frac{h}{2}\right) U_{n 1} \\
& U_{n 3}=F_{n}\left(x_{n}+h, y_{n}+h\right)+h k\left(x_{n}+h, y_{n}+h, x_{n}+\frac{h}{2}, y_{n}+\frac{h}{2}\right) U_{n 2} \\
& U_{n 4}=F_{n}\left(x_{n}+h, y_{n}+h\right)+\frac{h}{6}\left[k\left(x_{n}+h, y_{n}+h, x_{n}, y_{n}\right) U_{n 0}\right. \\
& +2 k^{2}\left(x_{n}+h, y_{n}+h, x_{n}+\frac{h}{2}, y_{n}+\frac{h}{2}\right)\left[U_{n 1}+U_{n 2}\right] \\
& \left.+k\left(x_{n}+h, y_{n}+h, x_{n}+h, y_{n}+h\right) U_{n 3}\right]
\end{aligned}
$$

The function $U_{n 4}(x, y)$ is unknown function, such that

$$
\begin{gathered}
F_{n}(x, y)=f(x, y)+\frac{h}{6} \sum_{i=0}^{n-1}\left[k\left(x, y, x_{i}, y_{i}\right) U_{i 0}+2 k\left(x, y, x_{i+1 / 2}, y_{i+1 / 2}\right)\left[U_{i 1}+U_{i 2}\right]\right. \\
+k\left(x, y, x_{i+1}, y_{i+1}\right) U_{i 3}
\end{gathered}
$$

where $F_{0}(x, y)=f(x, y), x_{i+1}=y_{i+1}=x_{i}+h, x_{i+1 / 2}=y_{i+1 / 2}=x_{i}+\frac{h}{2}$ for $0 \leq i \leq n-1, n=0,1, \ldots N$.

When $n=1$, the equation (12) becomes, 


$$
\begin{gathered}
U_{14}=F_{1}\left(x_{2}, y_{2}\right)+\frac{h}{6}\left[k\left(x_{2}, y_{2}, x_{1}, y_{1}\right) U_{10}+2 k\left(x_{2}, y_{2}, x_{1+1 / 2}, y_{1+1 / 2}\right)\left[U_{11}+U_{12}\right]\right. \\
\left.+k\left(x_{2}, y_{2}, x_{2}, y_{2}\right) U_{13}\right]
\end{gathered}
$$

Such that $F_{1}\left(x_{2}, y_{2}\right)=f\left(x_{2}, y_{2}\right)$, and

$U_{10}=f\left(x_{1}, y_{1}\right)$

$U_{11}=f\left(x_{1+1 / 2}, y_{1+1 / 2}\right)+\frac{h}{2} k\left(x_{1+1 / 2}, y_{1+1 / 2}, x_{1}, y_{1}\right) U_{10}$

$U_{12}=f\left(x_{1+1 / 2}, y_{1+1 / 2}\right)+\frac{h}{2} k\left(x_{1+1 / 2}, y_{1+1 / 2}, x_{1+1 / 2}, y_{1+1 / 2}\right) U_{11}$

$U_{13}=f\left(x_{2}, y_{2}\right)+h k\left(x_{2}, y_{2}, x_{1+1 / 2}, y_{1+1 / 2}\right) U_{12}$

We can written equation (12) as:

$$
\begin{gathered}
U_{n 4}=F_{n}\left(x_{n+1}, y_{n+1}\right)+\frac{h}{6}\left[k\left(x_{n+1}, y_{n+1}, x_{n}, y_{n}\right) U_{n 0}+2 k\left(x_{n+1}, y_{n+1}, x_{n+1 / 2}, y_{n+1 / 2}\right)\right. \\
\left.\left[U_{n 1}+U_{n 2}\right]+k\left(x_{n+1}, y_{n+1}, x_{n+1}, y_{n+1}\right) U_{n 3}\right]
\end{gathered}
$$

For $1 \leq n \leq N-1$, and

$$
\begin{aligned}
& U_{n 0}=U_{n-1,4} \\
& U_{n 1}=F_{n}\left(x_{n+1 / 2}, y_{n+1 / 2}\right)+\frac{h}{2} k\left(x_{n+1 / 2}, y_{n+1 / 2}, x_{n}, y_{n}\right) U_{n 0} \\
& U_{n 2}=F_{n}\left(x_{n+1 / 2}, y_{n+1 / 2}\right)+\frac{h}{2} k\left(x_{n+1 / 2}, y_{n+1 / 2}, x_{n+1 / 2}, y_{n+1 / 2}\right) U_{n 1} \\
& U_{n 3}=F_{n}\left(x_{n+1}, y_{n+1}\right)+h k\left(x_{n+1}, y_{n+1}, x_{n+1 / 2}, y_{n+1 / 2}\right) U_{n 2}
\end{aligned}
$$

Since the function $U_{n 4}=U_{n 4}(x, y)$ is the approximate solution at $\left(x_{n}, y_{n}\right)$ for equation (9).

\section{Block by block method:}

We suppose that the following linear two -dimensional Volterra integral equation:

$$
\mu u(x, y)=f(x, y)+\lambda \int_{0}^{x} \int_{0}^{y} k(x, y, t, s) u(t, s) d t d s
$$

has a unique solution . the idea behind B by BM is quite general, but is most easily understood by considering a specific case.

Use the Simpson's rule as a numerical integration formula, we get,

$$
u_{2} \square u\left(x_{2}, y_{2}\right)=f\left(x_{2}, y_{2}\right)+\frac{\lambda}{\mu} \int_{0}^{x} \int_{0}^{y} k\left(x_{2}, y_{2}, t, s\right) u(t, s) d t d s
$$

Approximating the integrals by Simpson's rule, if we knew $u_{1}(x, y)$, then we could compute $u_{2}(x, y)$ by

$$
\begin{gathered}
u_{2} \square u\left(x_{2}, y_{2}\right)=f\left(x_{2}, y_{2}\right)+\frac{h \lambda}{3 \mu}\left[k\left(x_{2}, y_{2}, t_{0}, s_{0}\right) u_{0}+4 k\left(x_{2}, y_{2}, t_{1}, s_{1}\right) u_{1}\right. \\
\left.+k\left(x_{2}, y_{2}, t_{2}, s_{2}\right) u_{2}\right]
\end{gathered}
$$

where $u_{0}=f\left(x_{0}, y_{0}\right)$.

Now, we have 


$$
u_{1} \square u\left(x_{1}, y_{1}\right)=f\left(x_{1}, y_{1}\right)+\frac{\lambda}{\mu} \int_{0}^{x} \int_{0}^{y} k\left(x_{1}, y_{1}, t, s\right) u(t, s) d t d s
$$

to evaluate the integrals on the right sides, we introduce another point $x_{1 / 2}=\frac{h}{2}$ and the corresponding value $u_{1 / 2}$ and use the Simpson's rulewith step size $h / 2$, then

$$
\begin{aligned}
u_{1}=f\left(x_{1}, y_{1}\right)+\frac{h \lambda}{6 \mu}\left[k\left(x_{1}, y_{1}, x_{0}, y_{0}\right) u_{0}\right. & +4 k\left(x_{1}, y_{1}, x_{1 / 2}, y_{1 / 2}\right) u_{1 / 2} \\
& \left.+k\left(x_{1}, y_{1}, x_{1}, y_{1}\right) u_{1}\right]
\end{aligned}
$$

where $u_{1 / 2}$ have unknown values, that can be estimated by Lagrange interpolation points $x_{0}, y_{0}, x_{1}, y_{1}, x_{2}, y_{2}$.

Therefore we obtain,

$$
u_{1 / 2}=\frac{3}{8} u_{0}+\frac{3}{4} u_{1}-\frac{1}{8} u_{2}
$$

Substituting from (3.25) into (3.24) we obtain:

$$
\begin{gathered}
u_{1}=f\left(x_{1}, y_{1}\right)+\frac{h \lambda}{6 \mu}\left[k\left(x_{1}, y_{1}, x_{0}, y_{0}\right) u_{0}+4 k\left(x_{1}, y_{1}, x_{1 / 2}, y_{1 / 2}\right)\left[\frac{3}{8} u_{0}+\frac{3}{4} u_{1}-\frac{1}{8} u_{2}\right]\right. \\
\left.+k\left(x_{1}, y_{1}, x_{1}, y_{1}\right) u_{1}\right]
\end{gathered}
$$

In equation (3.20) for $x \in[0, a]$ and $y \in[0, b]$, we divide $0=x_{0}<x_{1}<\ldots<x_{N}=a$, $0=y_{0}<y_{1}<\ldots<y_{N}=b$ be a partition of $[0, a],[0, b]$ with the step size $h$, such that, $x_{i}=i h, y_{i}=i h$ for $i, j=0,1, \ldots, N$.

Then we can construct B by BM, by setting $x=x_{2 m+1}, y=y_{2 m+1}$ to get,

$$
\begin{aligned}
u_{2 m+1}=f\left(x_{2 m+1}, y_{2 m+1}\right)+\frac{\lambda}{\mu}\left[\int_{0}^{x_{2 m}} \int_{0}^{y_{2 m}} k\left(x_{2 m+1}, y_{2 m+1}, t, s\right) u(t, s) d t d s\right. \\
\left.\quad+\int_{x_{2 m}}^{x_{2 m+1}} \int_{y_{2 m}}^{y_{2 m+1}} k\left(x_{2 m+1}, y_{2 m+1}, t, s\right) u(t, s) d t d s\right]
\end{aligned}
$$

Now, integration over $\left[0, x_{2 m}\right] \times\left[0, y_{2 m}\right]$ can be accomplished by Simpson's rule and the integral over $\left[x_{2 m}, x_{2 m+1}\right] \times\left[y_{2 m}, y_{2 m+1}\right]$ is computed by using a quadratic interpolation, so if

$u_{0} \square u\left(x_{0}, y_{0}\right)=f\left(x_{0}, y_{0}\right)$

We have,

$$
\begin{aligned}
u_{2 m+1}=f\left(x_{2 m+1}, y_{2 m+1}\right)+ & \frac{\lambda}{\mu}\left[\sum_{i=0}^{2 m} w_{i} k\left(x_{2 m+1}, y_{2 m+1}, x_{i}, y_{i}\right) u_{i}\right. \\
& +\frac{h}{6}\left[k\left(x_{2 m+1}, y_{2 m+1}, x_{2 m}, y_{2 m}\right) u_{2 m}\right. \\
& +4 k\left(x_{2 m+1}, y_{2 m+1}, x_{2 m+1 / 2}, y_{2 m+1 / 2}\right)\left[\frac{3}{8} u_{2 m}+\frac{3}{4} u_{2 m+1}-\frac{1}{8} u_{2 m+2}\right] \\
& \left.\left.+k\left(x_{2 m+1}, y_{2 m+1}, x_{2 m+1}, y_{2 m+1}\right) u_{2 m+1}\right]\right]
\end{aligned}
$$


Also, in a similar manner we have

$$
\begin{aligned}
& u_{2 m+2}=f\left(x_{2 m+2}, y_{2 m+2}\right)+\frac{\lambda}{\mu}\left[\int_{0}^{x_{2 m+2}} \int_{0}^{y_{2 m+2}} k\left(x_{2 m+2}, y_{2 m+2}, t, s\right) u(t, s) d t d s\right] \\
& u_{2 m+2}=f\left(x_{2 m+2}, y_{2 m+2}\right)+\frac{\lambda}{\mu} \sum_{i=0}^{2 m+2} w_{i} k\left(x_{2 m+2}, y_{2 m+2}, x_{i}, y_{i}\right) u_{i}
\end{aligned}
$$

where $\left\{w_{i}\right\}=\frac{1}{3}\{1,4,2, \ldots, 2,4,1\}, i=0,1, \ldots, 2 \mathrm{~m}$ and $x_{2 m+1 / 2}=x_{2 m}+\frac{h}{2}$,also $y_{2 m+1 / 2}=y_{2 m}+\frac{h}{2}$.

\section{Numerical Experiments and Discussions:}

\section{Example 1:}

Consider the linear two-dimensional Volterra integral equation:

$$
u(x, y)=x y-0.125 x^{5} y^{3}+\int_{0}^{x} \int_{0}^{y} x y s^{2} u(t, s) d t d s
$$

where the exact solution is $u(x, y)=x y$ and $0 \leq x, y \leq 1$, here $\lambda=1, \mu=1$. In table (5.1)-(5.3) we present the exact solution, the approximate numerical solutions and their corresponding errors for some points, we suppose that $N=20,50,80$.

\section{In tables (5.1)-(5.6):}

$u^{R . K} \rightarrow$ approximate solution of R. KM, $E^{R . K} \rightarrow$ the error of R. KM, $u^{\text {B.B }} \rightarrow$ approximate solution of B by BM and $E^{\text {B.B }} \rightarrow$ the error of B by BM .

Case 1: $N=20$,

\begin{tabular}{|l|l|c|c|c|c|c|}
\hline$x$ & $y$ & Exact sol. & $u^{R . K}$ & $E^{R . K}$ & $u^{B . B}$ & $E^{B . B}$ \\
\hline 0 & 0 & 0 & 0 & 0 & 0 & 0 \\
\hline 0.1 & 0.1 & 0.010000000 & 0.0100000188 & $1.880 \times 10^{-8}$ & 0.0100000195 & $1.959 \times 10^{-8}$ \\
\hline 0.2 & 0.2 & 0.040000000 & 0.0400022404 & $2.24048 \times 10^{-6}$ & 0.0400022467 & $2.24673 \times 10^{-6}$ \\
\hline 0.3 & 0.3 & 0.090000000 & 0.0900355494 & $3.55494 \times 10^{-5}$ & 0.0900355702 & $3.55702 \times 10^{-5}$ \\
\hline 0.4 & 0.4 & 0.160000000 & 0.1602460300 & $2.46030 \times 10^{-4}$ & 0.1602460742 & $2.46074 \times 10^{-4}$ \\
\hline 0.5 & 0.5 & 0.250000000 & 0.2510777921 & $1.07779 \times 10^{-3}$ & 0.2510778341 & $1.07783 \times 10^{-3}$ \\
\hline 0.6 & 0.6 & 0.360000000 & 0.3635284441 & $3.52844 \times 10^{-3}$ & 0.3635282581 & $3.52825 \times 10^{-3}$ \\
\hline 0.7 & 0.7 & 0.490000000 & 0.4994353363 & $9.43533 \times 10^{-3}$ & 0.4994339204 & $9.43392 \times 10^{-3}$ \\
\hline 0.8 & 0.8 & 0.640000000 & 0.6617454824 & $2.17454 \times 10^{-2}$ & 0.6617393619 & $2.17393 \times 10^{-2}$ \\
\hline 0.9 & 0.9 & 0.810000000 & 0.8547527831 & $4.47527 \times 10^{-2}$ & 0.8547316022 & $4.47316 \times 10^{-2}$ \\
\hline 1.0 & 1.0 & 1.000000000 & 1.084359749 & $8.43597 \times 10^{-2}$ & 1.084295562 & $8.42955 \times 10^{-2}$ \\
\hline
\end{tabular}

Table(5.1)

Case 2: $N=50$,

\begin{tabular}{|c|l|c|c|c|c|c|}
\hline$x$ & $y$ & Exact sol. & $u^{R . K}$ & $E^{R . K}$ & $u^{B . B}$ & $E^{B . B}$ \\
\hline 0 & 0 & 0 & 0 & 0 & 0 & 0 \\
\hline
\end{tabular}




\begin{tabular}{|l|l|l|l|l|l|l|}
\hline 0.1 & 0.1 & 0.010000000 & 0.0100000187 & $1.875 \times 10^{-8}$ & 0.0100000187 & $1.877 \times 10^{-8}$ \\
\hline 0.2 & 0.2 & 0.040000000 & 0.0400022400 & $2.24007 \times 10^{-6}$ & 0.0400022402 & $2.24026 \times 10^{-6}$ \\
\hline 0.3 & 0.3 & 0.090000000 & 0.0900355476 & $3.55476 \times 10^{-5}$ & 0.0900355480 & $3.55480 \times 10^{-5}$ \\
\hline 0.4 & 0.4 & 0.160000000 & 0.1602460205 & $2.46020 \times 10^{-4}$ & 0.1602460210 & $2.46021 \times 10^{-4}$ \\
\hline 0.5 & 0.5 & 0.250000000 & 0.2510777310 & $1.07773 \times 10^{-3}$ & 0.2510777242 & $1.07772 \times 10^{-3}$ \\
\hline 0.6 & 0.6 & 0.360000000 & 0.3635281038 & $3.52810 \times 10^{-3}$ & 0.3635280535 & $3.52805 \times 10^{-3}$ \\
\hline 0.7 & 0.7 & 0.490000000 & 0.4994338048 & $9.43380 \times 10^{-3}$ & 0.4994335491 & $9.43354 \times 10^{-3}$ \\
\hline 0.8 & 0.8 & 0.640000000 & 0.6617397087 & $2.17397 \times 10^{-2}$ & 0.6617387294 & $2.17387 \times 10^{-2}$ \\
\hline 0.9 & 0.9 & 0.810000000 & 0.8547338211 & $4.47338 \times 10^{-2}$ & 0.8547305011 & $4.47305 \times 10^{-2}$ \\
\hline 1.0 & 1.0 & 1.000000000 & 1.084303663 & $8.43036 \times 10^{-2}$ & 1.084293778 & $8.42937 \times 10^{-2}$ \\
\hline
\end{tabular}

\section{Table(5.2)}

Case 3: $N=80$,

\begin{tabular}{|l|l|c|c|c|c|c|}
\hline$x$ & $y$ & Exact sol. & $u^{R . K}$ & $E^{R . K}$ & $u^{B . B}$ & $E^{B . B}$ \\
\hline 0 & 0 & 0 & 0 & 0 & 0 & 0 \\
\hline 0.1 & 0.1 & 0.010000000 & 0.0100000187 & $1.875 \times 10^{-8}$ & 0.0100000187 & $1.876 \times 10^{-8}$ \\
\hline 0.2 & 0.2 & 0.040000000 & 0.0400022400 & $2.24009 \times 10^{-6}$ & 0.0400022401 & $2.24011 \times 10^{-6}$ \\
\hline 0.3 & 0.3 & 0.090000000 & 0.0900355476 & $3.55476 \times 10^{-5}$ & 0.0900355476 & $3.55476 \times 10^{-5}$ \\
\hline 0.4 & 0.4 & 0.160000000 & 0.1602460199 & $2.46019 \times 10^{-4}$ & 0.1602460197 & $2.46019 \times 10^{-4}$ \\
\hline 0.5 & 0.5 & 0.250000000 & 0.2510777255 & $1.07772 \times 10^{-3}$ & 0.2510777224 & $1.07772 \times 10^{-3}$ \\
\hline 0.6 & 0.6 & 0.360000000 & 0.3635280690 & $3.52806 \times 10^{-3}$ & 0.3635280491 & $3.52804 \times 10^{-3}$ \\
\hline 0.7 & 0.7 & 0.490000000 & 0.4994336440 & $9.43364 \times 10^{-3}$ & 0.4994335469 & $9.43354 \times 10^{-3}$ \\
\hline 0.8 & 0.8 & 0.640000000 & 0.6617390932 & $2.17390 \times 10^{-2}$ & 0.6617387155 & $2.17387 \times 10^{-2}$ \\
\hline 0.9 & 0.9 & 0.810000000 & 0.8547317752 & $4.47317 \times 10^{-2}$ & 0.8547305073 & $4.47305 \times 10^{-2}$ \\
\hline 1.0 & 1.0 & 1.000000000 & 1.084297548 & $8.42975 \times 10^{-2}$ & 1.084293738 & $8.42937 \times 10^{-2}$ \\
\hline
\end{tabular}

Table(5.3)

\section{Example 2:}

Consider the linear two-dimensional Volterra integral equation:

$$
u(x, y)=x \sin (\mathrm{y})+\frac{1}{4} x^{2}\left(x^{3} \cos (\mathrm{y})-\sin ^{2}(y)-x^{3}\right)+\int_{0}^{x} \int_{0}^{y}\left(x t^{2}+\cos (s)\right) u(t, s) d t d s(29)
$$

where the exact solution is $u(x, y)=x \sin y$ and $0 \leq x, y \leq 1$, here $\lambda=1, \mu=1$. In table (5.4)-(5.6) we present the exact solution, the approximate numerical solutions and their corresponding errors for some points, we suppose that $N=20,50,80$.

Case 1: $N=20$,

\begin{tabular}{|l|l|l|l|l|l|l|}
\hline$x$ & $y$ & Exact sol. & $u^{R . K}$ & $E^{R . K}$ & $u^{B . B}$ & $E^{B . B}$ \\
\hline
\end{tabular}




\begin{tabular}{|c|c|l|l|l|l|l|}
\hline 0 & 0 & 0 & 0 & 0 & 0 & 0 \\
\hline 0.1 & 0.1 & 0.009983341 & 0.0102985626 & $3.152209 \times 10^{-4}$ & 0.0102984714 & $3.151297 \times 10^{-4}$ \\
\hline 0.2 & 0.2 & 0.039733866 & 0.0420948398 & $2.360973 \times 10^{-3}$ & 0.0420947296 & $2.360863 \times 10^{-3}$ \\
\hline 0.3 & 0.3 & 0.088656062 & 0.0960620236 & $7.405961 \times 10^{-3}$ & 0.0960620397 & $7.405977 \times 10^{-3}$ \\
\hline 0.4 & 0.4 & 0.155767336 & 0.1720136585 & $1.624632 \times 10^{-2}$ & 0.1720139211 & $1.624658 \times 10^{-2}$ \\
\hline 0.5 & 0.5 & 0.239712769 & 0.2690460130 & $2.933324 \times 10^{-2}$ & 0.2690463848 & $2.933361 \times 10^{-2}$ \\
\hline 0.6 & 0.6 & 0.338785484 & 0.3857321589 & $4.694667 \times 10^{-2}$ & 0.3857318235 & $4.694633 \times 10^{-2}$ \\
\hline 0.7 & 0.7 & 0.450952381 & 0.5202947182 & $6.934233 \times 10^{-2}$ & 0.5202914295 & $6.933904 \times 10^{-2}$ \\
\hline 0.8 & 0.8 & 0.573884872 & 0.6706795493 & $9.679467 \times 10^{-2}$ & 0.6706682790 & $9.678340 \times 10^{-2}$ \\
\hline 0.9 & 0.9 & 0.704994218 & 0.8344513514 & $1.294571 \times 10^{-1}$ & 0.8344218264 & $1.294276 \times 10^{-1}$ \\
\hline 1.0 & 1.0 & 0.841470984 & 1.008420750 & $1.669497 \times 10^{-1}$ & 1.008353013 & $1.668820 \times 10^{-1}$ \\
\hline
\end{tabular}

Table(5.4)

Case 2: $N=50$,

\begin{tabular}{|c|c|c|c|c|c|c|}
\hline$x$ & $y$ & Exact sol. & $u^{R . K}$ & $E^{R . K}$ & $u^{B . B}$ & $E^{B . B}$ \\
\hline 0 & 0 & 0 & 0 & 0 & 0 & 0 \\
\hline 0.1 & 0.1 & 0.009983341 & 0.0102985675 & $3.152259 \times 10^{-4}$ & 0.0102985585 & $3.152168 \times 10^{-4}$ \\
\hline 0.2 & 0.2 & 0.039733866 & 0.0420948904 & $2.361024 \times 10^{-3}$ & 0.0420948957 & $2.361029 \times 10^{-3}$ \\
\hline 0.3 & 0.3 & 0.088656062 & 0.0960622119 & $7.406149 \times 10^{-3}$ & 0.0960622480 & $7.406185 \times 10^{-3}$ \\
\hline 0.4 & 0.4 & 0.155767336 & 0.1720140502 & $1.624671 \times 10^{-2}$ & 0.1720141263 & $1.624678 \times 10^{-2}$ \\
\hline 0.5 & 0.5 & 0.239712769 & 0.2690464557 & $2.933368 \times 10^{-2}$ & 0.2690465658 & $2.933379 \times 10^{-2}$ \\
\hline 0.6 & 0.6 & 0.338785484 & 0.3857319293 & $4.694644 \times 10^{-2}$ & 0.3857319264 & $4.694644 \times 10^{-2}$ \\
\hline 0.7 & 0.7 & 0.450952381 & 0.5202918897 & $6.933950 \times 10^{-2}$ & 0.5202914557 & $6.933907 \times 10^{-2}$ \\
\hline 0.8 & 0.8 & 0.573884872 & 0.6706698505 & $9.678497 \times 10^{-2}$ & 0.6706681747 & $9.678330 \times 10^{-2}$ \\
\hline 0.9 & 0.9 & 0.704994218 & 0.8344260926 & $1.294318 \times 10^{-1}$ & 0.8344216258 & $1.294274 \times 10^{-1}$ \\
\hline 1.0 & 1.0 & 0.841470984 & 1.008363084 & $1.668920 \times 10^{-1}$ & 1.008352641 & $1.668816 \times 10^{-1}$ \\
\hline
\end{tabular}

Table(5.5)

Case 3: $N=80$,

\begin{tabular}{|c|c|c|c|c|c|c|}
\hline$x$ & $y$ & Exact sol. & $u^{R . K}$ & $E^{R . K}$ & $u^{B . B}$ & $E^{B . B}$ \\
\hline 0 & 0 & 0 & 0 & 0 & 0 & 0 \\
\hline 0.1 & 0.1 & 0.009983341 & 0.0102985680 & $3.152263 \times 10^{-4}$ & 0.0102985679 & $3.152262 \times 10^{-4}$ \\
\hline 0.2 & 0.2 & 0.039733866 & 0.0420948963 & $2.361030 \times 10^{-3}$ & 0.0420948994 & $2.361033 \times 10^{-3}$ \\
\hline 0.3 & 0.3 & 0.088656062 & 0.0960622346 & $7.406172 \times 10^{-3}$ & 0.0960622486 & $7.406186 \times 10^{-3}$ \\
\hline 0.4 & 0.4 & 0.155767336 & 0.1720140993 & $1.624676 \times 10^{-2}$ & 0.1720141308 & $1.624679 \times 10^{-2}$ \\
\hline 0.5 & 0.5 & 0.239712769 & 0.2690465172 & $2.933374 \times 10^{-2}$ & 0.2690465585 & $2.933378 \times 10^{-2}$ \\
\hline
\end{tabular}




\begin{tabular}{|l|l|l|l|l|l|l|}
\hline 0.6 & 0.6 & 0.338785484 & 0.3857319254 & $4.694644 \times 10^{-2}$ & 0.3857319289 & $4.694644 \times 10^{-2}$ \\
\hline 0.7 & 0.7 & 0.450952381 & 0.5202916079 & $6.933922 \times 10^{-2}$ & 0.5202914398 & $6.933905 \times 10^{-2}$ \\
\hline 0.8 & 0.8 & 0.573884872 & 0.6706688137 & $9.678394 \times 10^{-2}$ & 0.6706681726 & $9.678329 \times 10^{-2}$ \\
\hline 0.9 & 0.9 & 0.704994218 & 0.8344233212 & $1.294291 \times 10^{-1}$ & 0.8344215878 & $1.294273 \times 10^{-1}$ \\
\hline 1.0 & 1.0 & 0.841470984 & 1.008356670 & $1.668856 \times 10^{-1}$ & 1.008352634 & $1.668816 \times 10^{-1}$ \\
\hline
\end{tabular}

Table(5.6)

\section{The Conclusion:}

From the previous discussions we conclude the following:

1) As $x$ and $y$ is increasing in $[0,1] \times[0,1]$, the errors due to block by block method and Runge-Kutta method are also increasing.

2) As $N$ is increasing, the errors are decreasing.

3) The errors due to the block by block method less than the errors due to the Runge-Kutta method.(i.e. block by block method better than Runge-Kutta method for solving linear Two-Dimensional Volterra integral equation with continuous kernel).

\section{References:}

[1] A. J. Jerri, Introduction to Integral Equations with Applications, John Wiley and Sons, INC(1999).

[2] P.Linz, Analytic and Numerical Methods for Volterra Equations, SIAM, Philadelphia, 1985.

[3] C. T. H. Baker, H. Geoffrey, F. Miller, Treatment of Integral Equations by Numerical Methods, Acad. Press, 1982.

[4] L. M. Delves and J. L. Mohamed, Computational Methods for Integral Equations, Cambridge, 1985.

[5] K. E. Atkinson, A Survey of Numerical Method for the Solution of Fredholm Integral Equation of the Second Kind, Philadelphia, 1976.

[6] K. E. Atkinson, The Numerical Solution of Integral Equation of the Second Kind, Cambridge, 1997.

[7] M. A. Golberg. ed, Numerical Solution of Integral Equations, Boston, 1990.

[8] R. Katani, S. Shahmorad, Block by Block Method for The System of Nonlinear Volterra Integral Equations, App. Math, 34(2010)400-406.

[9] M. M. EL-Borai, M. A. Abdou and M. M. EL-Kojok, On a discussion of nonlinear integral equation of type VolterraFredholm, J. KSIAM, Vol. 10, No.2, 59-83, 2006.

[10] H. Guoqiang, K. Hayami, K. Sugihura, and W. Jiong, Extrapolation method of iterated collocation solution for twodimensional nonlinear Volterra integral equations, J. Appl. Math. Comput. 112 (2000) 49-61.

[11] H. Guoqiang, W.Jiong, Extrapolation of Nystrom solution for two dimensional nonlinear Fredholm integral equations, J.Comput. Appl. Math. 134 (2001) 259-268.

[12] M. A. Abdou, Fredholm-Volterra integral equation of the first kind and contact problem. Appl. Math. Comput. 125 (2002)177-193.

[13] I. L. El-Kalla and A. M. Al-Bugami, Adomian and Block-by-Block Methods to Solve Nonlinear Two-Dimensional Volterra Integral Equation, AJBAS, 6(3) (2011) 335-340. 